\title{
Research on Financial Data Query and Distribution Scheme Based on SQL Database
}

\author{
Xiaolan Fu \\ School of Economics and Trade Tourism, Hefei Technology College, Hefei 230011, China \\ Correspondence should be addressed to Xiaolan Fu; pengyongfang@hgu.edu.cn
}

Received 30 August 2020; Revised 10 October 2020; Accepted 6 November 2020; Published 26 November 2020

Academic Editor: Hongju Cheng

Copyright ( 2020 Xiaolan Fu. This is an open access article distributed under the Creative Commons Attribution License, which permits unrestricted use, distribution, and reproduction in any medium, provided the original work is properly cited.

\begin{abstract}
With the advance of optimization and merger colleges and universities, a university often contains more than one campus. The traditional centralized education management system has been unable to meet the needs of use. The model detects the intrusion by dividing the clusters in the clustering result into normal clusters and abnormal clusters and analyzing the weighted average density of object $x$ to be detected in each cluster and the weighted overlapping distance of $x$ and each centre point. We verified the intrusion detection performance of the model on the KDD Cup 99 dataset. The experimental results show that the model established in this paper has certain theoretical value.
\end{abstract}

\section{Introduction}

The educational administration information system of colleges and universities mainly manages the relevant educational administration information of colleges and universities. Relying on the basic platform of campus network, it plays an increasingly important role in teaching and management application $[1,2]$. By combining intrusion detection algorithms, this paper solves the problems of $k$ -mode algorithm based on rough set theory and applies the improved algorithm to intrusion detection [3]. Firstly, aiming at $k$ pattern clustering in initial class centre selection, an initial class centre selection algorithm unweights based on weighted density and weighted overlap distance is proposed. Secondly, this article further improves the model on the basis of the above [4].

As an important part of China's education, intrusion detection helps systems respond to cyberattacks by monitoring and analyzing user and system activity and statistically analyzing system vulnerabilities, detecting known intrusions and alarms, auditing new anomalous behaviors, and assessing integrity of critical data files. System administrators' security management capabilities have been extended through auditing and monitoring, intrusion identification and response, reducing the workload of system administra- tors [3]. In addition, the intrusion detection system dilutes the restrictions on professionals in network security, making it easy for nonprofessional personnel to manage network systems. In addition, the intrusion detection system can respond to the discovered network intrusion, illegal operation, timely and proactive response and management [5].

\section{Related Work}

Database technology management is about the existing problems of intrusion detection systems. Data mining technology has been widely used in the field of intrusion detection in recent years. Among them, cluster-based unsupervised intrusion detection has attracted extensive attention. At present, many kinds of clustering algorithms have been applied to intrusion detection [6]. As an effective extension of $k$ -means algorithm, it can effectively process classified data. It inherits the characteristics of efficient $k$-means algorithm. The algorithm to implement has been widely applied in many fields. Therefore, $K$ pattern algorithm has a very broad application prospect in the field of intrusion detection. However, there are few researches on applying $K$ pattern [7].

At present, it is in a difficult existing problem of intrusion detection systems. Data mining technology has been widely used in the field of intrusion detection in recent years. 
Among them, cluster-based unsupervised intrusion detection has attracted extensive attention. At present, many kinds of clustering algorithms have been applied to intrusion detection. As an effective extension of $k$-means algorithm, it can effectively process classified data. It inherits the often cannot handle the concurrent operation efficiently [8,9]. Distributed practical optimal allocation algorithm ion detection helps systems respond to cyberattacks by monitoring and analyzing user and system activity. In order to count and analyze system vulnerabilities and detect known intrusions and alarms, this article adopts the audit strategy. With characteristics of efficiency, the algorithm is simple and easy to implement, which has been widely applied in many fields. Therefore, $K$ pattern algorithm has a very broad application prospect in the field of intrusion detection. However, there are few researches on applying $K$ pattern [10].

\section{Methodology}

3.1. Distributed Database Data Distribution Algorithm. Distributed database refers to the use of high-speed computer network which will be physically dispersed multiple data storage units [11], which is a proactive and proactive network security defense strategy that can make up for the shortcomings of traditional static security policies and thus becomes a reasonable complement to traditional static defense strategies such as firewalls [12]. Intrusion detection is to detect known intrusions and alarms by monitoring and analyzing user and system activities and by counting and analyzing system vulnerabilities [13]. In addition, the model also helps the system respond to cyberattacks by reviewing new abnormal behaviors and evaluating the integrity of key data files [14]. The traditional centralized database performs data query operations based on SQL language. However, in distributed database, query operations need to take into account a number of factors, and the performance of query programs in different schemes varies greatly. This paper proposes and studies ion detection, which conducts statistics and analysis of system vulnerabilities by monitoring and analyzing user and system activities. In addition, this mode can help the system respond to network attacks by detecting known intrusions and alarms and considering the relevance during the audit. The administrator's security management functions will include the following permissions, auditing and monitoring [15]. In addition, intrusion identification and response have been expanded, thereby reducing the workload of system administrators; SQL has its advantages of high scalability and high concurrency [16].

System databases have data distribution; suppose $S$ is mathematical allocation, then $S=\left(S_{1}, S_{2}, \cdots, S_{m}\right)$, which are sites and sites connected to each other through the network. The set of program transactions running on the network is set to $T=\left(T_{1}, T_{2}, \cdots, T\right)_{n}$, as a basis for the operation of the program transaction $[17,18]$. The dataset on the network is set to $D$, then $D=\left(D_{1}, D_{2}, \cdots, D_{p}\right)$. Then, the problem that the data distribution algorithm needs to deal with translates into designing an algorithm $D_{i}$, a reasonable copy of the site in different $S$ configurations so that the overall system achieves the highest total performance, the total cost to a minimum, and the program at this time marked $C\left(D_{i}, S_{j}\right.$, $\left.T_{k}\right)[19,20]$. The allocation clusters have performed the allocation, in which communication costs within need to be defined and clarified. In addition, for the above definition, the internal equation is included as shown in $[21,22]$

$$
\begin{gathered}
\operatorname{Arg} C_{i}=\frac{\sum_{i=1}^{n} \sum_{j=1 \wedge \neq j}^{n} \operatorname{CC}\left(S_{i}, S_{j}\right)}{n *(n-1)}, \\
\operatorname{ArgCC}\left(C_{i}, C_{j}\right)=\frac{\sum_{i=1}^{m} \sum_{j=1 \wedge \neq j}^{i=1} \operatorname{CC}\left(S_{i}, S_{j}\right)}{m * n} .
\end{gathered}
$$

The total communication cost of all the clusters in the system can be defined as

$$
\mathrm{AllACC}=\frac{\sum_{i=1}^{n} \sum_{j=1}^{n} \operatorname{ACC}\left(C_{i}, C_{j}\right)}{n * n} .
$$

The above cost to optimize the allocation is based on genetic algorithm data $[23,24]$. For the principle of genetic algorithm research and application, many related references are discussed in detail; the length of the space is not discussed in detail [25]. The improvement of only allocation is shown in Figure 1; Figure 1 is reproduced from Liu et al. [26].

The method of generating of chromosomes and adopted some project will use to make the initial population cover as much as possible, improving the probability of finding the optimal solution, improving chromosome selection strategy, and adding the optimal selection algorithm [27]. The traditional genetic algorithm uses the method of roulette, which may result in the loss of some excellent chromosomes [28]. This problem is solved by adding the traditional centralized database that performs data query operations based on SQL language. However, in distributed database, query operations need to take into account a number of factors, and the performance of query programs in different schemes varies greatly [29]. This article proposes and studies ion detection, which monitors and analyses user and system activities and counts and analyzes system vulnerabilities [30]. In addition, the relevant model established in this article can detect known intrusions and alarms and conduct audits. At the same time, we also consider the relevant best selection algorithm to help the system respond to network attacks. Dynamic changes in the probability of crossover and mutation are not only to ensure the generation rate of new chromosomes to ensure the diversity of chromosomes but also to ensure that good chromosomes are not damaged. The algorithm is not easy to fall into the local optimum [31]. The determination of the dynamic probability is related to the change of fitness. The definitions of chromosomes and fitness functions are given in [32].

3.2. College Teaching Management Distributed Database Platform Data Processing Technology Model. The data input function is involved in many subsystems of the system. It is the realization process of the basic energy that the educational system normally uses. The main process is that the user inputs the data through the system manually; the file is read and analyzed automatically. The Chinese database management system network downloads and uses other ways to get the data and saves it to the database. For the set, the data 


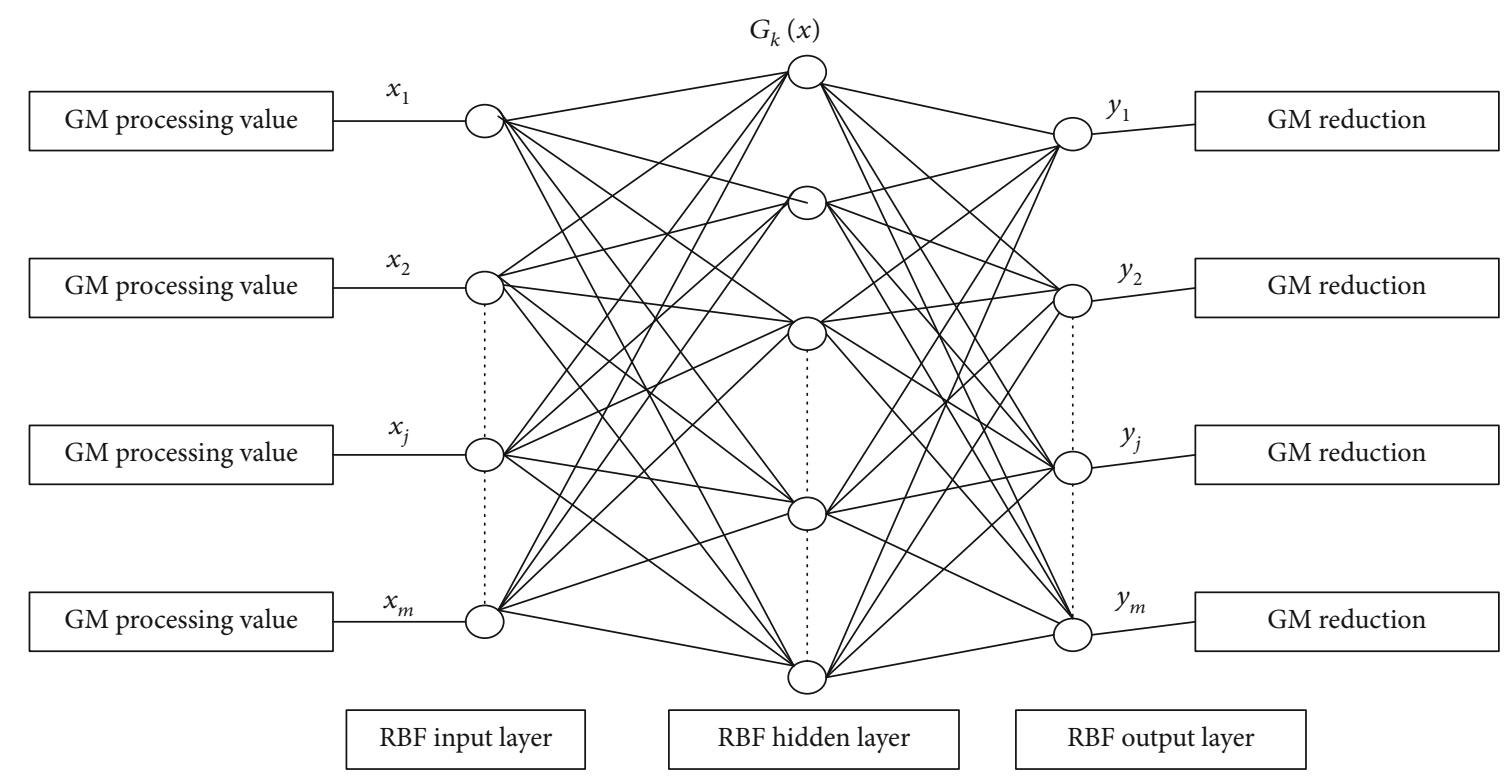

FIGURE 1: The processing of genetic algorithm.

will be saved directly to the database table, and for distributed database systems, you also need to consider the data allocation problem. In the above, we designed a data distribution algorithm based on clustering and genetic algorithm in distributed database $[33,34]$.

In this section, we discuss the implementation and application of data allocation algorithm with practical application. Theoretically, the aspects to be considered in calculating the cost after data allocation include the cost of transaction processing and the cost of data storage [35]. However, the cost of data storage is negligible due to the low data storage cost at present, and only the cost of transaction processing is considered. In the above, we studied the problem of communication cost after clustering. In fact, the data operation needs to be considered in data distribution, including the query cost and the cost of local and remote update. The cost of a local query is calculated as the product of the average search cost within the cluster and the average number of searches for that cluster, multiplied by the data size, which is shown in [36]

$$
\begin{aligned}
\operatorname{CLRsum}\left(T_{k}, D_{i}, C_{j}\right)= & \operatorname{CLR}\left(T_{k}, D_{i}, C_{j}\right) * \operatorname{Size}\left(D_{i}\right), \\
\operatorname{CLUsum}\left(T_{k}, D_{i}, C_{j}\right)= & \operatorname{CLU}\left(T_{k}, D_{i}, C_{j}\right) * \operatorname{Count} U \\
& \cdot\left(T_{k}, D_{i}, C_{j}\right) * \operatorname{Size}\left(D_{i}\right), \\
\operatorname{CLUsum}\left(T_{k}, D_{i}, C_{j}\right)= & \sum_{k=1}^{m} \sum_{i=1}^{n} \sum_{j=1}^{p} \operatorname{CLU}\left(T_{k}, D_{i}, C_{j}\right) \\
& * \operatorname{Count} U\left(T_{k}, D_{i}, C_{j}\right) * \operatorname{Size}\left(D_{i}\right) .
\end{aligned}
$$

The cost of remote communication sympathy detection can be achieved by monitoring and analyzing user and system activities. At the same time, in order to conduct statistics and analysis of system vulnerabilities, we will detect known intru-
TABle 1: Prediction error of different models in the result.

\begin{tabular}{lcc}
\hline $\begin{array}{l}\text { Record } \\
\text { number } \\
\text { (million) }\end{array}$ & $\begin{array}{c}\text { Centralized storage } \\
\text { asked (seconds) }\end{array}$ & $\begin{array}{c}\text { Distributed database } \\
\text { storage asked (seconds) }\end{array}$ \\
\hline 1 & 0.4 & 0.7 \\
10 & 8.5 & 3.1 \\
20 & 38.0 & 4.8 \\
\hline
\end{tabular}

sions and alerts and audit Nally. In addition, we will multiply the data size to help the system respond to cyberattacks. The relevant mathematical principles are shown in

$$
\begin{aligned}
& \mathrm{CRC}_{\text {sum }}\left(\mathrm{T}_{\mathrm{k}}, \mathrm{D}_{\mathrm{i}}, \mathrm{C}_{\mathrm{j}}\right)=\sum_{k=1}^{m} \sum_{i=1}^{n} \sum_{j=1}^{p} U \operatorname{rate}\left(T_{k}, D_{i}, C_{j}\right) \\
& * \operatorname{CRC}\left(T_{k}, D_{i}, C_{j}\right) * \operatorname{Size}\left(D_{i}\right), \\
& \operatorname{Cost}\left(T_{k}, D_{i}, C_{j}\right)= \operatorname{CLR}_{\text {sum }}\left(T_{k}, D_{i}, C_{j}\right)+\operatorname{CLU}_{\text {sum }} \\
& \cdot\left(T_{k}, D_{i}, C_{j}\right)+\mathrm{CRU}_{\text {sum }}\left(T_{k}, D_{i}, C_{j}\right) \\
&+\mathrm{CRC}_{\text {sum }}\left(T_{k}, D_{i}, C_{j}\right) .
\end{aligned}
$$

The research of distributed database data allocation algorithm based on genetic algorithm is to minimize the cost of data processing. That is to say, minimizing equation (5) to achieve this goal, we first use the above formula for calculating the cost of communication between sites to separate different sites into a cluster. In the educational management information system, the division of clusters is relatively simple, because the geographical differences between different campuses in the school are more obvious. In other words, divide a campus into a cluster, or divide a teacher into a cluster. Then, according to the steps of the genetic algorithm, equation (5) is used as the fitness function. 


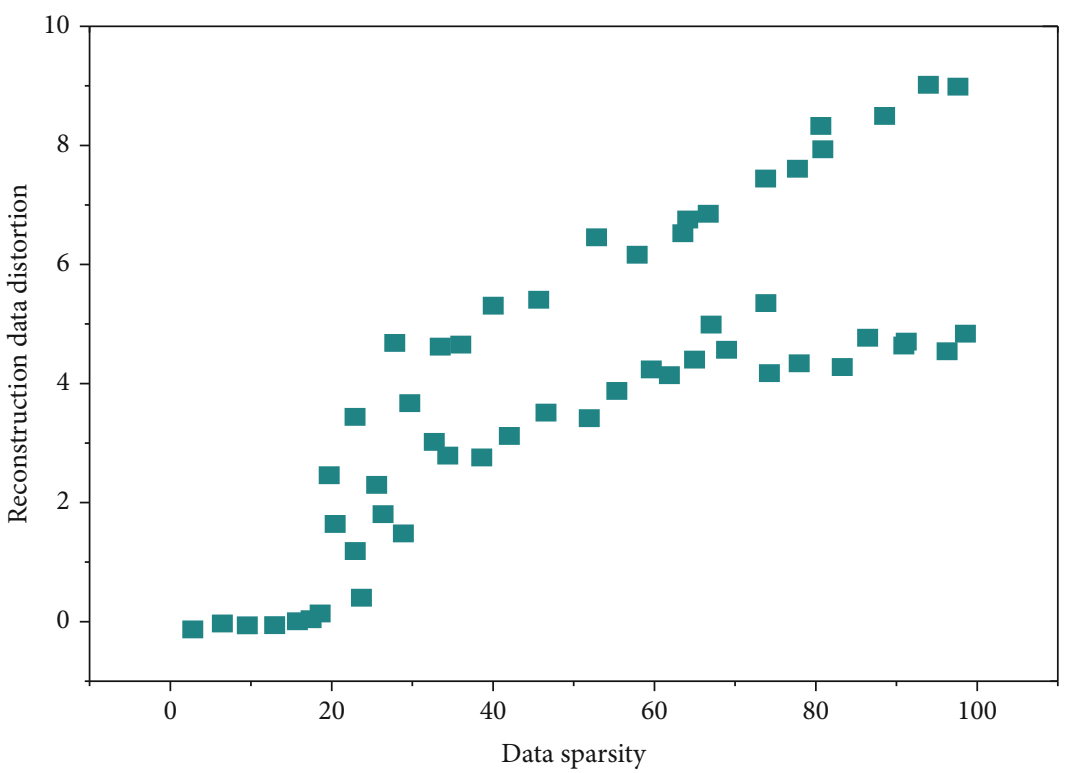

Figure 2: Fitting curve of parameter identification.

TABLE 2: Prediction error of different models.

\begin{tabular}{lcccc}
\hline Error & Linear regression model & Model 1 & Model 2 & Model 3 \\
\hline Maximum relative error $\left(E_{\text {mic }}\right)(\%)$ & 41.6 & 38.8 & 26.2 & 13.3 \\
Average relative error $\left(E_{\text {ave }}\right)(\%)$ & 5.1 & 13.3 & 5.8 & 3.7 \\
Root mean square error $(\mathrm{RMSE})$ & 8.9 & 8.0 & 7.3 & 4.1 \\
\hline
\end{tabular}

\section{Result Analysis and Discussion}

According to the above test results, the system has effectively improved and shortened it, realizing the effectiveness of the system. During viewing the existing problems of intrusion detection systems, data mining technology has been widely used in the field of intrusion detection in recent years. Among them, cluster-based unsupervised intrusion detection has attracted extensive attention. At present, many kinds of clustering algorithms have been applied to intrusion detection. Table 1 is reproduced from Liu et al. (2019).

As an effective extension of $k$-means algorithm, $K$-mode algorithm can be effective but often cannot handle the concurrent operation efficiently. Distributed practical optimal allocation algorithm is detection which helps systems respond to cyberattacks by monitoring and analyzing user and system activity and statistically analyzing system vulnerabilities. The traditional centralized database detects known intrusions and alarms in the design, usually adopts the audit process, and performs data query operations based on the SQL language.

However, in distributed database, query operations need to take into account a number of factors, and the performance of query programs in different schemes varies greatly. This paper proposes and studies ion detection through monitoring and analysis of user and system activities. In addition, in order to complete the task of counting and analyzing system vulnerabilities, we will detect consistent intrusions and alerts and will further complete audits and consider reclassified data to help
TABLE 3: Distributed database optimization query method comparison experiment.

\begin{tabular}{lcc}
\hline $\begin{array}{l}\text { Number of } \\
\text { queries } \\
(10000)\end{array}$ & $\begin{array}{c}\text { General distributed } \\
\text { database query asked } \\
\text { (seconds) }\end{array}$ & $\begin{array}{c}\text { Optimize the distribution } \\
\text { of database query asked } \\
\text { (seconds) }\end{array}$ \\
\hline 1 & 0.2 & 0.3 \\
10 & 4.7 & 1.2 \\
20 & 10.9 & 4.8 \\
\hline
\end{tabular}

the system respond to cyberattacks. It inherits the characteristics of being efficient. The algorithm to implement has been widely applied in many fields. Therefore, $K$ pattern algorithm has a very broad application prospect in the field of intrusion detection. However, there are few researches on applying $K 1$. The fitting curve of parameter identification is shown in Figure 2. The centralized database has less storage time to optimize the department within a certain amount of time, but the centralized database and time operation are not the time of the centralized database storage. Table 2 lists the prediction error results of different models.

Experiments show warehousing applying data allocation optimization algorithm in which obviously all the data are stored with attention. At present, many kinds of clustering algorithms have been applied to intrusion detection. As an effective extension, it usually cannot handle concurrent operations efficiently. The best distribution algorithm for 


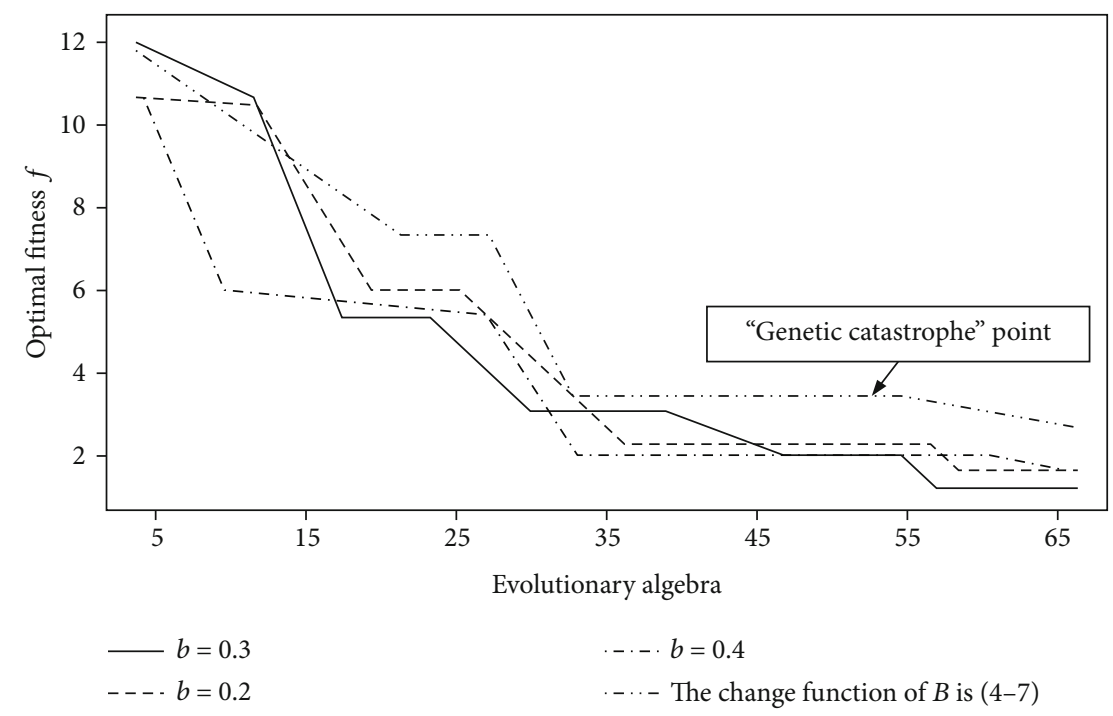

FIgURE 3: Diagram of the relationship between evolutionary algebra and optimal fitness.

distributed practical use is ion detection, which can help the system respond to cyberattacks by monitoring and analyzing user and system activities. In addition, in order to complete the statistics and analysis of system vulnerabilities, we will detect known intrusions and alert. At the same time, we will adopt audit methods, $k$-means algorithm, and $K$-mode algorithm to effectively process classified data. It inherits related data slowly, and it also performs a series of related operations.

In addition, we use statistics and analysis of system vulnerabilities to detect known intrusions and alerts. At the same time, we will further adopt auditing, using an optimized average speed. The distributed database optimization query method comparison experiment is shown in Table 3.

In data query, the traditional centralized database performs data query operations based on SQL language. However, in distributed database, query operations need to take into account a number of factors, and the performance of query programs in different schemes varies greatly. This paper proposes and studies ion detection which helps systems respond to cyberattacks by monitoring and analyzing user and system activity. At the same time, we will conduct statistics and analysis of system vulnerabilities and detect known intrusions and alerts. In order to conduct a further review and consider relevant factors, we will give the best solution at the end to greatly reduce the occurrence of related error rates.

This article is to verify the probability and mutation probability. The above probability coefficients are compared through experiments, and the optimal selection operator is given below. In addition, we will obtain the results by using different probability coefficient values. For further observations, we found that when $a=0.9$, the evolution is very fast, but it quickly enters premature development, becoming a local optimum. It is impossible to conduct an effective search. When $b=0.2$, the change is small; it is local and inextricable. The related test results and analysis are shown in Figure 3. Figure 3 and Table 1 are reproduced from Liu et al. (2019).
Through research, we found that when $b=0.4$ in the relevant index, the index has too much change, so it cannot be eliminated. Through further research, it is found that related measures can eliminate certain effects, but this affects the speed of evolution. Only when $a=0.8$ and $b=0.3$ can there be high-speed evolution, and it is not easy to enter the "premature" search efficiently and stable state. $f$ is the minimum value of the overall fitness function, and $f$ is the fitness value that usually cannot effectively handle concurrent operations. The best distribution algorithm for distributed practical use is ion detection, which can monitor and analyze user and system activities. At the same time, we will further carry out relevant statistics and analysis to prevent system vulnerabilities. In order to detect known intrusions and alarms and audits used by individuals to help the system respond to network attacks, comparison of database indicators for different research methods is shown in Figure 4.

Through further analysis, we found that the research on Figure 4 also jumped out of the premature state with changes in adaptability. We found that in order to make the search wider, we can learn to find better results. In order to further understand that niche operators and sorting preference operators are a better algorithm, the text needs to further compare the two algorithms. In order to conduct a further review and consider relevant factors, we will give the best solution at the end to greatly reduce the occurrence of related error rates.

\section{Conclusions}

Distributed database system used in various industries in the production and life of society is the basis for data processing applications. The distributed database system is used in various industries in the production and life of the society, and it is the basis of data processing applications. In this paper, a centralized management system in multicampus university teaching system applications exists in data redundancy; query efficiency is low and so on. Distributed database technology is used to build educational system basic data 


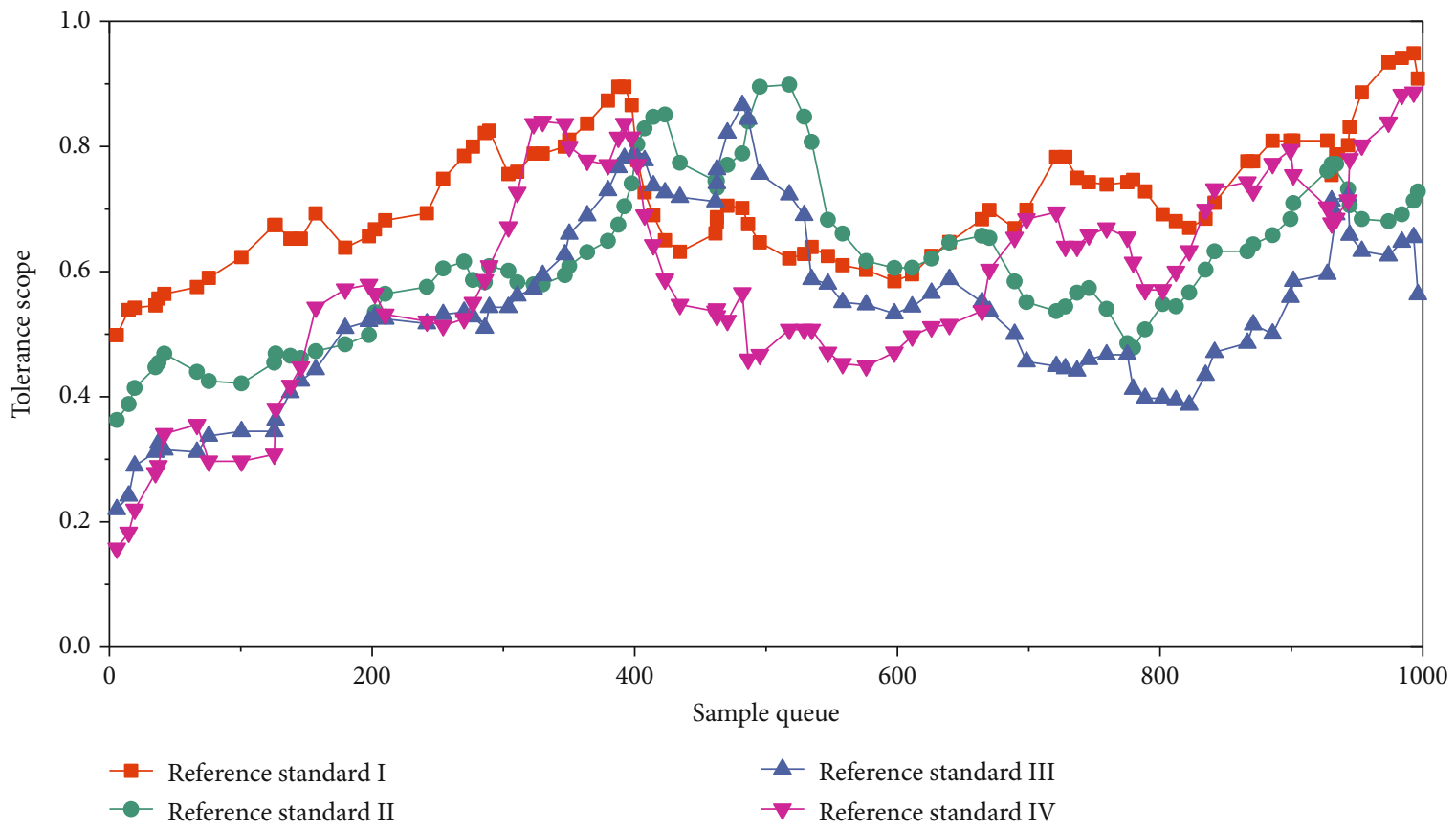

FIGURE 4: Comparison of database indicators for different research methods.

platform for the program, with the use of distributed database reliable high sex.

High operating efficiency and other features are used to solve the multicampus college database construction issues. In-depth study of the application of the teaching system is done in colleges and universities; MySQL database management system is used to achieve a concrete realization of a distributed database. In addition, in order to adapt to related needs, we need to analyze the functions and performance of the system. On this basis, we established the functions and logical and physical deployment models of the school's administrative management information system and designed the system's data architecture scheme. Through analysis, we believe that the use of Java language in the development environment concretely realizes the main functional blocks of the system. In addition, the focus of related research in this article is to realize data by implementing the main functions and supporting functions of the system for the application of the education system. Finally, the test case verifies the system functions and performance indicators to meet actual needs.

\section{Data Availability}

All trial data is available online.

\section{Conflicts of Interest}

The author declares no conflicts of interest.

\section{Acknowledgments}

This work is supported by the Anhui Provincial Quality Engineering Project of colleges and universities in 2018 (2018ylzy130), Key Research Projects of Humanities and Social Sciences in Universities of Anhui Province in 2019 (SK2019A0941), and Anhui Province 2020 university outstanding talents support plan project.

\section{References}

[1] B. Neelon, A. J. O'Malley, and V. A. Smith, "Modeling zeromodified count and semicontinuous data in health services research part 1: background and overview," Statistics in Medicine, vol. 35, no. 27, pp. 5070-5093, 2016.

[2] F. Dexter and R. H. Epstein, "Fifteen years of research on surgical case duration prediction by combining preoperatively available service and surgeon data," Journal of the American College of Surgeons, vol. 229, no. 6, pp. 633-634, 2019.

[3] K. J. Ryan, M. S. Hamada, and S. B. Vardeman, "Estimating a service-life distribution based on production counts and a failure database," Journal of Quality Technology, vol. 49, no. 2, pp. 172-185, 2017.

[4] T. R. Tsai, Y. Lio, N. Jiang, Y. J. Lin, and Y. Y. Fan, "Economical sampling plans with warranty based on truncated data from burr type xii distribution," Journal of the Operational Research Society, vol. 66, no. 9, pp. 1511-1518, 2017.

[5] B. Neelon, A. J. O'Malley, and V. A. Smith, "Modeling zeromodified count and semicontinuous data in health services research part 2: case studies," Statistics in Medicine, vol. 35, no. 27, pp. 5094-5112, 2016.

[6] J. Qi, S. Li, Y. Gao, K. Yang, and P. Liu, "Joint optimization model for train scheduling and train stop planning with passengers distribution on railway corridors," Journal of the Operational Research Society, vol. 69, no. 4, pp. 556-570, 2018.

[7] H. Cho and J. Lee, "Searching for logistics and regulatory determinants affecting overseas direct purchase: An empirical cross-national study," The Asian Journal of Shipping and Logistics, vol. 33, no. 1, pp. 11-18, 2017. 
[8] L. I. Hongping, S. Zhiwu, L. I. Hao, G. Jing, W. U. Chunxia, and X. U. Xiang, Research on estrogenicity distribution and cleanup during sewage treatment process, Environmental Science \& Technology, 2016.

[9] Z. Cao and H. Jiang, "Research on scattering models of air particles with variable size distribution and shape distribution," Applied Optics, vol. 58, no. 13, p. 3370, 2019.

[10] K. Granville and S. Drekic, "On a 2-class polling model with reneging and \$\$ k_i\$ k i -limited service," Annals of Operations Research, vol. 274, no. 1-2, pp. 267-290, 2019.

[11] X. H. Zhang, P. Feng, J. R. Xu, L. B. Feng, and S. Qing, "Numerical research on combining flue gas recirculation sintering and fuel layered distribution sintering in the iron ore sintering process," Energy, vol. 192, p. 116660, 2020.

[12] L. Franceschini, D. H. M. D. Vieira, A. C. Zago, R. K. Azevedo, V. D. Abdallah, and R. J. da Silva, "New data on myxobolus imparfinis (cnidaria, myxosporea): host, distribution, and ultrastructural morphology," Parasitology Research, vol. 118, no. 6, pp. 1967-1973, 2019.

[13] Z. Lin-Lian, C. Shu-Qin, C. Mei-Ting, and Z. M. Bureau, Research on offshore wave prediction based on wind and wave data obtained in Zhoushan in the past years, Coastal Engineering, 2018.

[14] G. Wang, A. Gunasekaran, and E. W. T. Ngai, "Distribution network design with big data: model and analysis," Annals of Operations Research, vol. 270, no. 1-2, pp. 539-551, 2018.

[15] C. Li, A. Ingersoll, M. Janssen et al., "The distribution of ammonia on Jupiter from a preliminary inversion of Juno microwave radiometer data," Geophysical Research Letters, vol. 44, no. 11 , pp. 5317-5325, 2017.

[16] P. Cao, T. Miwa, and T. Morikawa, "Use of probe vehicle data to determine joint probability distributions of vehicle location and speed on an arterial road," Transportation Research Record: Journal of the Transportation Research Board, vol. 2421, no. 1, pp. 103-114, 2014.

[17] L. Jiancheng, W. Chunyong, Y. Wei, and L. Zhenhua, "Research on the ranging statistical distribution of laser radar with a constant fraction discriminator," IET Optoelectronics, vol. 12, no. 2, pp. 114-117, 2018.

[18] J. J. Liang, A. Belcastro, and J. Levi, "Sex distribution and sex data handling in published otolaryngology research," The Laryngoscope, vol. 129, no. 12, 2019.

[19] F. G. Cozman and D. D. Mauá, "On the semantics and complexity of probabilistic logic programs," Journal of Artificial Intelligence Research, vol. 60, pp. 221-262, 2017.

[20] V. G. Guerra, A. E. Achiles, and R. Béttega, "Influence of droplet size distribution on liquid dispersion in a venturi scrubber: experimental measurements and cfd simulation," Industrial \& Engineering Chemistry Research, vol. 56, no. 8, pp. 2177-2187, 2017.

[21] V. Reniers, D. Van Landuyt, A. Rafique, and W. Joosen, "Object to nosql database mappers (ondm): a systematic survey and comparison of frameworks," Information Systems, vol. 85, no. Nov., pp. 1-20, 2019.

[22] R. Chopade and V. K. Pachghare, "Ten years of critical review on database forensics research," Digital Investigation, vol. 29, pp. 180-197, 2019.

[23] A. Fabregat, F. Korninger, G. Viteri et al., "Reactome graph database: efficient access to complex pathway data," PLoS Computational Biology, vol. 14, no. 1, article e1005968, 2018.
[24] G. A. Schreiner, D. Duarte, and R. dos Santos Mello, "Bringing sql databases to key-based nosql databases: a canonical approach," Computing, vol. 102, no. 1, pp. 221-246, 2020.

[25] Y. H. Hsiao, M. C. Chen, and W. C. Liao, "Logistics service design for cross-border E-commerce using Kansei engineering with text-mining-based online content analysis," Telematics and Informatics, vol. 34, no. 4, pp. 284-302, 2017.

[26] S. Harikumar and M. R. Kaimal, "Subspacedb: in-database subspace clustering for analytical query processing," Data \& Knowledge Engineering, vol. 121, pp. 109-129, 2019.

[27] Y. Sun, Y. Wang, and H. Yang, "Bidirectional database storage and sql query exploiting rram-based process-in-memory structure," ACM Transactions on Storage, vol. 14, no. 1, pp. 1-19, 2018.

[28] A. Sander and R. Wauer, "Integrating terminologies into standard sql: a new approach for research on routine data," Journal of Biomedical Semantics, vol. 10, no. 1, p. 7, 2019.

[29] H. K. Khanuja and D. Adane, "Monitor and detect suspicious transactions with database forensic analysis," Journal of Database Management, vol. 29, no. 4, pp. 28-50, 2018.

[30] G. Hui, W. Xuan, L. Li, G. Wenxian, L. Chunjin, and F. Binghua, The reseach of the face recognition system based on matlab, Electronics World, 2018.

[31] Y. Liu, M. Ma, X. Liu, N. Xiong, A. Liu, and Y. Zhu, "Design and analysis of probing route to defense sink-hole attacks for Internet of Things security," IEEE Transactions on Network Science and Engineering, vol. 7, no. 1, pp. 356-372, 2020.

[32] F. Long, N. Xiong, A. V. Vasilakos, L. T. Yang, and F. Sun, “A sustainable heuristic QoS routing algorithm for pervasive multi-layered satellite wireless networks," Wireless Networks, vol. 16, no. 6, pp. 1657-1673, 2010.

[33] Y. Zhou, D. Zhang, and N. Xiong, "Post-cloud computing paradigms: a survey and comparison," Tsinghua Science and Technology, vol. 22, no. 6, pp. 714-732, 2017.

[34] W. Pan and C. Chai, "Measuring software stability based on complex networks in software," Cluster Computing, vol. 22, no. S2, pp. 2589-S2598, 2019.

[35] W. Pan and C. Chai, "Structure-aware Mashup service clustering for cloud-based Internet of Things using genetic algorithm-based clustering algorithm," Future Generation Computer Systems, vol. 87, pp. 267-277, 2018.

[36] G. Yang, Q. Yang, and H. Jin, "A novel trust recommendation model for mobile social network based on user motivation," Electronic Commerce Research, vol. 5, no. 7, pp. 145-160, 2019. 